\title{
MATCHING TO GANTRIES FOR MEDICAL SYNCHROTRONS
}

\author{
M. Benedikt, C. Carli, CERN/PS , CH-1211 Geneva 23, Switzerland
}

\section{Abstract}

Treatment of tumours by hadron-therapy is greatly improved if the patient can be irradiated from different directions. This task is performed by a gantry, i.e. a section of beam line that can be rotated around the patient. The gantry optics have to be designed in such a way that the beam at the patient is independent of the rotation angle. The various matching techniques are briefly reviewed in the light of the current development in medical synchrotrons towards active scanning, which requires a small, high-precision beam spot at the patient. In particular, beam delivery systems with rotators are discussed.

\section{INTRODUCTION}

In a medical machine the designer has the problem of matching the beam coming from the accelerator to the rotating gantry, as shown in Figure 1.

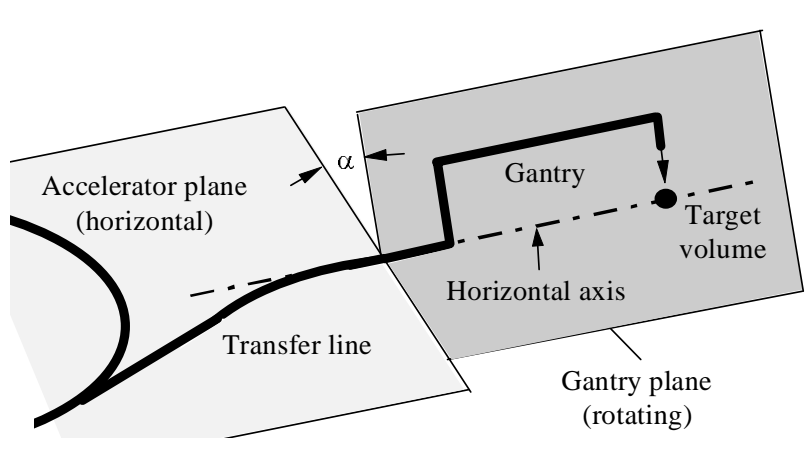

Figure 1: Schematic view of a rotating gantry.

The following requirements have to be fulfilled independent of the rotation angle:

- Constant spot size and shape at the treatment volume.

- No correlation between momentum and position.

- No change of the beam optics inside the gantry.

There appear to be only two methods, that satisfy all the above requirements, and a third method (one to one gantry) that partially fulfills them.

Consider a vector $\mathbf{y}$, containing the transverse phase-space coordinates of a particle. The statistical average of any distribution of particles in phase space is then given by the $\sigma$-matrix [1] defined as

$$
\sigma=\left\langle\mathbf{y} \mathbf{y}^{\mathrm{T}}\right\rangle,
$$

where the brackets mean the expectation value. For an uncoupled beam, all elements coupling the horizontal and the vertical phase space vanish and the $\sigma$-matrix is of the form shown below,

$$
\sigma_{u c}=\left(\begin{array}{cccc}
\left\langle x^{2}\right\rangle & \left\langle x x^{\prime}\right\rangle & 0 & 0 \\
\left\langle x^{\prime} x\right\rangle & \left\langle x^{\prime 2}\right\rangle & 0 & 0 \\
0 & 0 & \left\langle z^{2}\right\rangle & \left\langle z z^{\prime}\right\rangle \\
0 & 0 & \left\langle z^{\prime} z\right\rangle & \left\langle z^{\prime 2}\right\rangle
\end{array}\right) .
$$

With the definition of the statistical emittance,

$$
E_{x}=\sqrt{\left\langle x^{2}\right\rangle\left\langle x^{\prime 2}\right\rangle-\left\langle x x^{\prime}\right\rangle^{2}}
$$

the relationship between the $\sigma$-matrix and the Twiss formalisms is found as,

$$
\gamma_{x}=\frac{\left\langle x^{\prime 2}\right\rangle}{E_{\mathrm{x}}}, \quad \alpha_{\mathrm{x}}=-\frac{\left\langle x x^{\prime}\right\rangle}{E_{\mathrm{x}}}, \quad \beta_{\mathrm{x}}=\frac{\left\langle x^{2}\right\rangle}{E_{\mathrm{x}}}
$$

\section{SYMMETRIC BEAM METHOD}

In the symmetric beam method, the gantry is matched directly to the fixed beam line coming from the accelerator. To obtain the same beam behaviour inside the gantry and at the patient, independent of rotation, the beam has to be symmetric at the gantry entrance. The necessary symmetry conditions can be derived by the use of the $\sigma$-matrix. For an uncoupled beam the $\sigma$-matrix at the end of the fixed beam line, just in front of the gantry is given by:

$$
\sigma=\left(\begin{array}{cccc}
E_{\mathrm{x}} \beta_{\mathrm{x}} & -E_{\mathrm{x}} \alpha_{\mathrm{x}} & 0 & 0 \\
-E_{\mathrm{x}} \alpha_{\mathrm{x}} & E_{\mathrm{x}} \gamma_{\mathrm{x}} & 0 & 0 \\
0 & 0 & E_{\mathrm{z}} \beta_{\mathrm{z}} & -E_{\mathrm{z}} \alpha_{\mathrm{z}} \\
0 & 0 & -E_{\mathrm{z}} \alpha_{\mathrm{z}} & E_{\mathrm{z}} \gamma_{\mathrm{z}}
\end{array}\right),
$$

and after a rotation, $\mathbf{R}$, the new $\sigma$-matrix is given by

$$
\sigma_{2}=\mathbf{R} \sigma_{1} \mathbf{R}^{\mathrm{T}}
$$

When the incoming beam is rotationally symmetric, the matrix $\sigma_{2}$ will be independent of the rotation angle. Therefore the following constraints have to be fulfilled at the entry to the gantry:

$$
E_{x} \beta_{x}=E_{z} \beta_{z} \quad E_{x} \alpha_{x}=E_{z} \alpha_{z} \quad E_{x} \gamma_{x}=E_{z} \gamma_{z},
$$


which means that the following beam- and optics properties are required:

- Equal emittances $E_{x}=E_{z}$.

- Equal Twiss parameters $\beta_{x}=\beta_{z}, \alpha_{x}=\alpha_{z}, \gamma_{x}=\gamma_{z}$.

- Zero dipersion function $D=0, D^{\prime}=0$.

Thus the beam must be symmetric in real space and of identical shape in the two transverse phase spaces. It is not sufficient to have only a physically 'round' beam at the gantry entrance. To have equal Twiss parameters in both planes is not very limiting in practice but the constraint of equal emittances is a severe problem for resonant extraction from a synchrotron. Therefore this method is best suited for a cyclotron based facility.

\section{ONE TO ONE GANTRY METHOD}

For this method, the gantry must be a $1: 1$ or $1:-1$ mapping, achromatic structure, with phase advances being multiples of $\pi$ for both transverse planes. For simplicity $\mu_{\mathrm{x}}=\mu_{\mathrm{z}}=2 \pi$ is considered. The transfer matrix for the gantry is the $4 \times 4$ unit matrix I. The gantry rotation is described with a rotation matrix $\mathbf{R}_{\alpha}$. The overall matrix $\mathbf{M}_{0}$, from the end of the fixed beam line to the treatment volume, is then given by:

$$
\mathbf{M}_{0}=\mathbf{M}_{\text {gantry }} \mathbf{R}_{\alpha}=\mathbf{I R}_{\alpha}=\left(\begin{array}{cccc}
\cos \alpha & 0 & \sin \alpha & 0 \\
0 & \cos \alpha & 0 & \sin \alpha \\
-\sin \alpha & 0 & \cos \alpha & 0 \\
0 & -\sin \alpha & 0 & \cos \alpha
\end{array}\right)
$$

Thus, the beam seen from the gantry, is rotated at the treatment volume by the negative gantry angle $-\alpha$. This rotation cannot be avoided but if the beam at the gantry entrance is symmetric in real space, the patient will always 'see' the same particle distribution. Therefore

$$
E_{x} \beta_{x}=E_{z} \beta_{z} \quad \text { and } \quad D=0, D^{\prime}=0
$$

are required at the gantry entrance. The disadvantage of the one-to-one gantry method is that, as long as the beam does not fulfill all the conditions of the symmetric beam method, the beam is coupled inside the gantry, resulting in changing beam envelopes.

\section{ROTATOR METHOD}

In the rotator method [2] an insertion containing only quadrupoles is placed just in front of the gantry. This section of beam line (the rotator) is then rotated in proportion to the gantry rotation.

\subsection{Basic principle}

Consider a section of bending-free transfer line with betatron phase advances of $2 \pi$ in the horizontal and $\pi$ in the vertical plane with a transfer matrix of the form

$$
\mathbf{M}_{r o t}=\left(\begin{array}{cccc}
1 & 0 & 0 & 0 \\
0 & 1 & 0 & 0 \\
0 & 0 & -1 & 0 \\
0 & 0 & 0 & -1
\end{array}\right)
$$

If this line is physically rotated by half the gantry angle $(\alpha / 2)$ the overall transfer matrix $\mathbf{M}_{0}$ from the end of the fixed beam line through the rotator to the gantry entrance is obtained as,

$$
\mathbf{M}_{0}=\mathbf{R}_{\alpha / 2} \mathbf{M}_{R o t} \mathbf{R}_{\alpha / 2}=\mathbf{M}_{R o t} .
$$

The final overall transfer matrix maps the incoming normal modes directly to those of the gantry without any cross-coupling and independent of the gantry angle.

$$
\left(\begin{array}{c}
x \\
x^{\prime} \\
z \\
z^{\prime}
\end{array}\right)_{\text {Gantry }}=\left(\begin{array}{cccc}
1 & 0 & 0 & 0 \\
0 & 1 & 0 & 0 \\
0 & 0 & -1 & 0 \\
0 & 0 & 0 & -1
\end{array}\right)\left(\begin{array}{c}
x \\
x^{\prime} \\
z \\
z^{\prime}
\end{array}\right)_{\text {Input }} .
$$

The dispersion function $\left(D, D^{\prime}\right)$ is automatically rotated and matched at the same time, since in a bending-free region, the dispersion function acts like a betatron oscillation. This can be used for a simplified gantry design (i.e. fewer quadrupole magnets, shorter gantry). Additional advantages are:

- No requirements on the beam symmetry at the gantry entrance.

- Dispersion at the gantry entrance can be finite.

- Uncoupled beam inside the gantry.

The rotator method is best suited for a slow extracted beam from a synchrotron that has unequal emittances in the transverse planes.

\subsection{Rotator design}

In the above theory, the only visible constraint on the design of rotators is that the phase advances must be $2 \pi$ and $\pi$. However, one has to be aware that the overall transfer matrix does not contain any information about beam sizes and chromatic effects inside the structures. A rotation by $\pi / 2$ is equivalent to a change from focusing to defocusing in the rotator and therefore FODO structures lead to very large fluctuations of the Twiss functions and beam sizes due to the opposite signs of $\alpha_{x}$ and $\alpha_{z}$ at the entrance. For this reason, rotators should be designed with approximately equal Twiss parameters for the transverse planes at the entrance, which ensures a controlled beam behaviour at any rotation angle. Figure 2 shows a design example, the structure consists of two doublets that match the equal Twiss functions at the entrance $\left(\beta_{x}=\beta_{z}=4 \mathrm{~m}, \alpha_{\mathrm{x}}=\alpha_{\mathrm{z}}=0\right)$ into a FODO channel that gives the required phase advances of $\mu_{x}=2 \pi$ and $\mu_{z}=\pi$. 


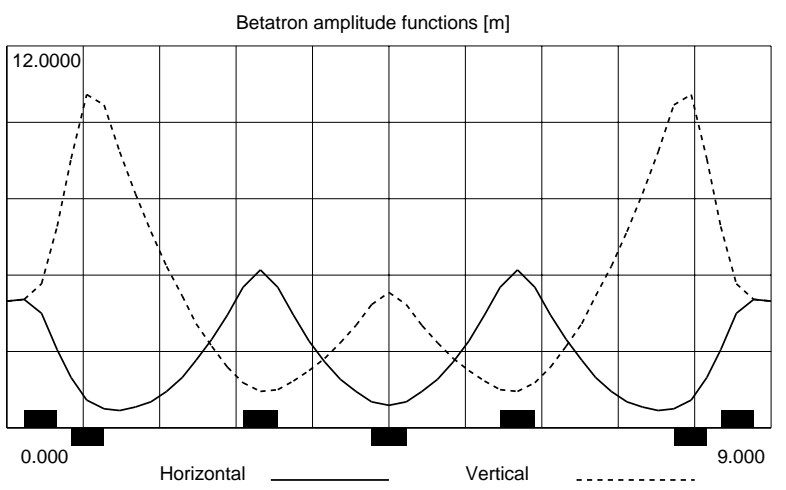

Figure 2: Twiss functions for Doublet FODO rotator.

\subsection{Extension modules}

To deliver beam to different gantries with only one rotator a modular beam delivery system can be used [3]. 'Extension modules' with optical properties similar to the rotator are inserted between the rotator and the different gantries. These modules are fixed in the accelerator plane. They must be achromatic sections of transfer line with phase advances of $n \cdot \pi$ in the transverse planes. Modules that provide a deflection must therefore have a closed dispersion bump. Figure 3 shows a $60^{\circ}$ deflecting extension module based on the Doublet FODO rotator.
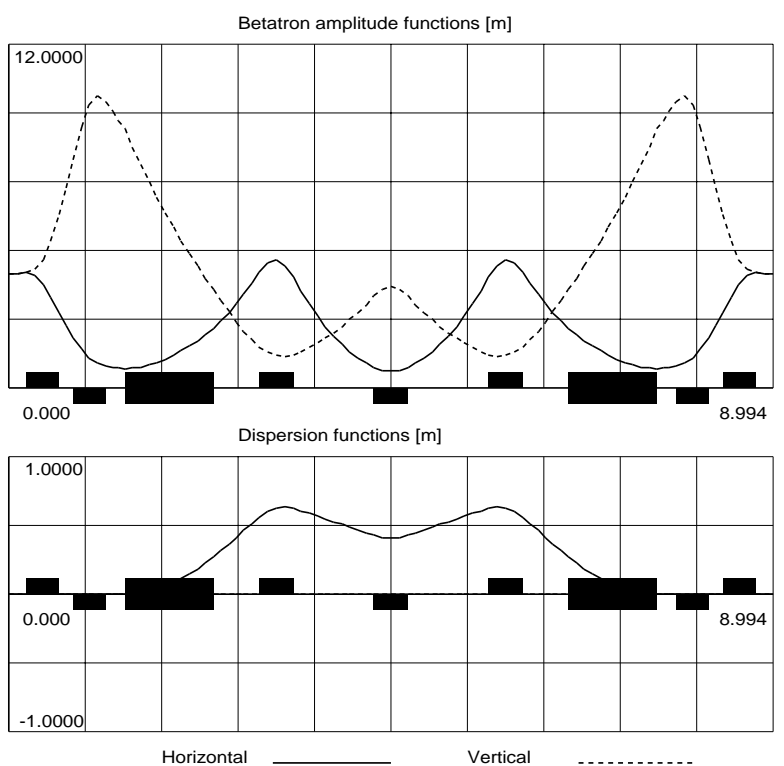

Figure 3: Extension module providing $60^{\circ}$ deflection

Two C-shaped dipoles have been inserted such that they give a closed dispersion bump. Powering the dipoles deflects the beam towards one gantry. To send the beam to a different gantry, the dipoles are not powered and the beam goes straight through the first dipole, where the structure is continued with the standard rotator lattice. Figure 4 illustrates the geometry, the first two quadrupoles are commonly used by the deflecting and the straight-through extension modules.

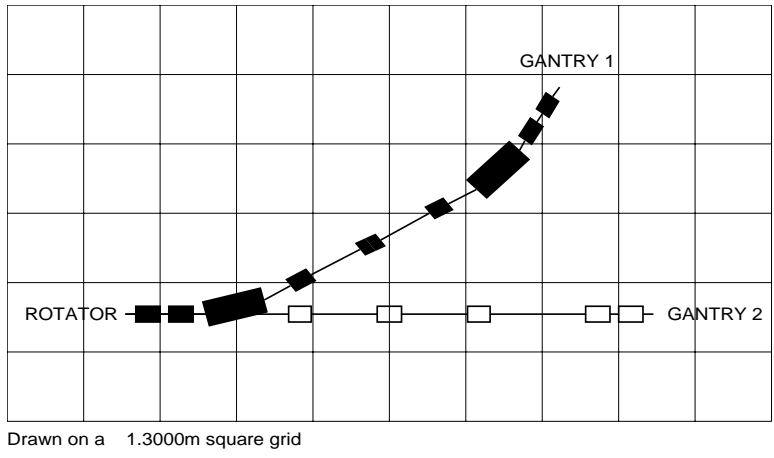

Figure 4: Geometry of deflection and straight-through extension modules.

\section{CONCLUSIONS}

In this paper the different techniques for matching therapy beams to gantries are reviewed. The methods can be summarised as follows:

Symmetric Beam Method:

- Requires a fully symmetric beam, i.e. equal emittances and Twiss functions at gantry entrance.

- The dispersion must be zero at the gantry entrance.

- Well suited for beams from cyclotrons.

One-to-one Gantry Method:

- Phase advances in the gantry must be multiples of $\pi$ in both transverse planes.

- Requires a symmetric beam in real space at the gantry entrance.

- The dispersion must be zero at the gantry entrance.

- Beam sizes change inside the gantry.

- Suited for beams from synchrotrons and cyclotrons, but non-perfect matching.

Rotator Method:

- Requires an additional quadrupole lattice section with phase advances of $2 \pi$ and $\pi$ in the transverse planes. This insertion (rotator) has to be rotated proportional to the gantry angle.

- In principle, no restrictions on the beam symmetry.

- In principle, no restrictions on the dispersion function which may simplify the gantry design.

- Well suited for beams from synchrotrons and cyclotrons.

\section{REFERENCES}

[1] J. Buon, A statistical description of a particle beam subjected to a linear and coupled betatron motion, LAL/RT 96-03.

[2] L. C. Teng, private communications, Fermilab, laboratory notebook (Jan. 1970), Oct. 1986.

[3] M. Benedikt, C. Carli, Optical design of a beam delivery system using a rotator, CERN/PS 96-41. 